\title{
Some comparison theorems and their applications in Finsler geometry
}

\author{
Songting Yin ${ }^{1,2}$, Qun $\mathrm{He}^{1 *}$ and Daxiao Zheng ${ }^{1}$
}

\section{"Correspondence:}

hequn@mail.tongji.edu.cn

'Department of Mathematics,

Tongji University, Siping Road,

Shanghai, China

Full list of author information is

available at the end of the article

\begin{abstract}
By using arbitrary volume forms, we establish Laplacian comparison theorems for Finsler manifolds under certain curvature conditions. As applications, some volume comparison theorems and Mckean type eigenvalue estimates of Finsler manifolds are obtained. Moreover, we also generalize Calabi-Yau's linear volume growth theorem, and Milnor's results on curvature and the fundamental group to the Finsler setting. MSC: 53C60; 53B40
\end{abstract}

Keywords: comparison theorem; Finsler-Laplacian; weighted Ricci curvature

\section{Introduction}

In recent years, Finsler geometry has developed rapidly in its global and analytic aspects. The present main work is to generalize and improve some famous theorems of the Riemann geometry to the Finsler setting. Among these issues, the Finsler-Laplacian is one of the most important and interesting projects. As is well known, there are several definitions of the Finsler-Laplacian, including the nonlinear Laplacian, the mean-value Laplacian and so on, in Finsler geometry. With regard to the nonlinear Finsler-Laplacian, some Laplacian comparison theorems, volume comparison theorems, and various estimations on the first eigenvalue have been established [1-5].

In [2], Shen first generalized comparison theorems to the Finsler geometry. Afterwards, $\mathrm{Wu}$ and Xin [3] proved Laplacian comparison theorems, volume comparison theorems under various flags, and Ricci and $S$-curvature conditions. Recently, using the Ricci curvature condition, and the distortion $\tau$ instead of $S$-curvature, $\mathrm{Wu}[4]$ and Zhao and Shen [5] further generalized volume comparison theorems in [2] and [3], respectively. It should be noted here that by utilizing the weighted Ricci curvature condition $\operatorname{Ric}_{N} \geq c$, Ohta and Sturm [1] and Ohta [6] gave another version of these theorems, which are more concise than the corresponding ones in [2] and [3].

In the Riemannian case, Ding [7] obtained a new Laplacian comparison theorem by the Ricci curvature condition Ric $\leq c<0$. Later, this result was generalized to Finsler manifolds in [3]. For a Finsler $n$-manifold with nonpositive flag curvature, if its Ricci curvature satisfies Ric $\leq c<0$, then the following holds whenever the distance function $\rho$ is smooth:

$$
\Delta \rho \geq \operatorname{ct}_{c}(\rho)-\|S\| .
$$

@2014 Yin et al.; licensee Springer. This is an Open Access article distributed under the terms of the Creative Commons Attribution License (http://creativecommons.org/licenses/by/2.0), which permits unrestricted use, distribution, and reproduction in any medium, provided the original work is properly cited. 
Here

$$
\operatorname{ct}_{c}(\rho)=\left\{\begin{array}{ll}
\sqrt{c} \cdot \cot (\sqrt{c} \rho) & c>0, \\
\frac{1}{\rho} & c=0, \\
\sqrt{-c} \cdot \operatorname{coth}(\sqrt{-c} \rho) & c<0,
\end{array} \quad\|S\|_{x}:=\sup _{X \in T_{x} M \backslash 0} \frac{|S(X)|}{F(X)} .\right.
$$

In this paper, we shall further improve this theorem by using the weighted Ricci curvature condition and remove the term of the $S$-curvature. To be precise, we will give the following result.

Theorem 1.1 Let $(M, F, d \mu)$ be a Finsler n-manifold with nonpositive flag curvature and nonpositive S-curvature. If the weighted Ricci curvature satisfies $\operatorname{Ric}_{n+1} \leq c$, then the following holds whenever the distance function $\rho$ is smooth:

$$
\Delta \rho \geq \operatorname{ct}_{c}(\rho)
$$

where $\mathrm{ct}_{c}(\rho)$ is defined by (1.1).

In addition to this, the Laplacian comparison theorem under the flag curvature and $S$ curvature condition is also obtained. As applications, we give some volume comparison theorems under the above-described conditions. It is worth mentioning that all the results we obtained are more concise than those in the related literature $[3,4]$ and more similar to the Riemannian case in form.

In $[8,9]$, Calabi and Yau stated that the volume of any complete noncompact Riemannian manifold with nonnegative Ricci curvature has at least linear growth. In [4], Wu has established the Finsler version of Calabi-Yau's linear volume growth theorem by using an extreme volume form. His result is

$$
\operatorname{vol}_{\max }\left(B_{p}(R)\right) \geq C(p) R,
$$

where vol $_{\max }$ denotes the volume with respect to the maximal volume form. In the present paper, we will further claim that for an arbitrary volume form Calabi-Yau's result still holds.

Theorem 1.2 Let $(M, F, d \mu)$ be a complete noncompact Finsler n-manifold with finite reversibility $\lambda$. If the weighted Ricci curvature satisfies $\operatorname{Ric}_{N} \geq 0, N \in(n, \infty)$, then

$$
\begin{aligned}
& \operatorname{vol}_{F}^{d \mu}\left(B_{p}^{+}(R)\right) \geq C\left(N, \lambda, \operatorname{vol}_{F}^{d \mu}\left(B_{p}^{+}(1)\right)\right) R \\
& \operatorname{vol}_{F}^{d \mu}\left(B_{p}^{-}(R)\right) \geq C\left(N, \lambda, \operatorname{vol}_{F}^{d \mu}\left(B_{p}^{-}(1)\right)\right) R
\end{aligned}
$$

where $B_{p}^{+}(R)$ (resp. $\left.B_{p}^{-}(R)\right)$ denotes the forward (resp. backward) geodesic ball of radius $R$ centered at $p$ and $C$ denotes the constant depending on $N, \lambda$, and $\operatorname{vol}_{F}^{d \mu}\left(B_{p}^{+}(1)\right)$ (resp. $\left.\operatorname{vol}_{F}^{d \mu}\left(B_{p}^{-}(1)\right)\right)$.

In Riemannian geometry, Mckean [10] proved that if $(M, g)$ is a complete and simply connected Riemannian $n$-manifold with sectional curvature $K \leq-a^{2}$, then the first eigenvalue $\lambda_{1}(M) \geq \frac{(n-1)^{2} a^{2}}{4}$. Afterwards, this result was extended by Ding in [7], stating that for a complete noncompact and simply connected Cartan-Hadamard manifold satisfying 
Ric $\leq-a^{2}$ the first eigenvalue $\lambda_{1}$ can be estimated below by $\frac{a^{2}}{4}$. A few years ago, these results were generalized to the Finsler setting by Wu and Xin [3]. In their paper, some conditions such as 'finite reversibility' and some restrictions on $S$-curvature should be satisfied, which are natural conditions in Finsler geometry and satisfied automatically in the Riemannian case.

In the present paper, we further generalize the Mckean type estimations to Finsler manifolds. We note that our results are as neat and simple as in the Riemannian case.

Theorem 1.3 Let $(M, F, d \mu)$ be a complete noncompact and simply connected Finsler nmanifold with finite reversibility $\lambda$ and nonpositive $S$-curvature. If the flag curvature satisfies $K \leq-a^{2}$, then

$$
\lambda_{1}(M) \geq \frac{(n-1)^{2} a^{2}}{4 \lambda^{2}}
$$

Theorem 1.4 Let $(M, F, d \mu)$ be a complete noncompact and simply connected Finsler $n$ manifold with finite reversibility $\lambda$, nonpositive flag curvature and nonpositive $S$-curvature. If the weighted Ricci curvature satisfies $\operatorname{Ric}_{n+1} \leq-a^{2}$, then

$$
\lambda_{1}(M) \geq \frac{a^{2}}{4 \lambda^{2}} .
$$

Remark 1.5 In Theorem 1.4, the condition 'nonpositive flag curvature' is necessary and it is a substitute for the condition 'Cartan-Hadamard manifold' in [7]. Since in the Riemannian case flag curvature is just sectional curvature, this condition is a natural condition.

Remark 1.6 The definitions of the reversibility $\lambda$ and $S$-curvature will be given in Sections 2,4 below. When $(M, F)$ is a Riemannian manifold, $\lambda=1, S=0$, and the above two results coincide with [10] and [7], respectively. Further, when $(M, F)$ is a Finsler manifold, the corresponding lower bounds obtained in [3] are $\lambda_{1}(M) \geq \frac{\left((n-1) a-\text { sup }_{M}\|S\|\right)^{2}}{4 \lambda^{2}}$ and $\lambda_{1}(M) \geq \frac{\left(a-\sup _{M}\|S\|\right)^{2}}{4 \lambda^{2}}$, respectively.

This paper is organized as follows. In Section 2, the related fundamentals of Finsler geometry such as Finsler metric, weighted Ricci curvature, gradient vector, FinslerLaplacian, and some lemmas are briefly introduced. The main results will be proved in Sections 3, 4, 5, respectively.

\section{Preliminaries}

Let $M$ be an $n$-dimensional smooth manifold and $\pi: T M \rightarrow M$ be the natural projection from the tangent bundle $T M$. Let $(x, y)$ be a point of $T M$ with $x \in M, y \in T_{x} M$, and let $\left(x^{i}, y^{i}\right)$ be the local coordinates on $T M$ with $y=y^{i} \frac{\partial}{\partial x^{i}}$. A Finsler metric on $M$ is a function $F: T M \rightarrow[0,+\infty)$ satisfying the following properties:

(i) Regularity: $F(x, y)$ is smooth in $T M \backslash 0$.

(ii) Positive homogeneity: $F(x, \lambda y)=\lambda F(x, y)$ for $\lambda>0$.

(iii) Strong convexity: The fundamental quadratic form

$$
g:=g_{i j}(x, y) d x^{i} \otimes d x^{j}, \quad g_{i j}:=\frac{1}{2}\left[F^{2}\right]_{y^{i} y^{j}}
$$

is positively definite. 
Let $\mathcal{U} \subset M$ be an open set and $V=v^{i} \frac{\partial}{\partial x^{i}}$ be a nonzero vector field on $\mathcal{U}$. Define

$$
\begin{aligned}
& g_{V}(X, Y):=X^{i} Y^{j} g_{i j}(x, V), \quad \forall X=X^{i} \frac{\partial}{\partial x^{i}}, Y=Y^{i} \frac{\partial}{\partial x^{i}}, \\
& D_{\frac{\partial}{\partial x^{i}}}^{V} \frac{\partial}{\partial x^{j}}:=\Gamma_{i j}^{k}(x, V) \frac{\partial}{\partial x^{k}},
\end{aligned}
$$

where $\Gamma_{i j}^{k}(x, V)$ are Chern connection coefficients. Then

$$
\begin{aligned}
& D_{X}^{V} Y-D_{Y}^{V} X=[X, Y], \\
& X g_{V}(Y, Z)=g_{V}\left(D_{X}^{V} Y, Z\right)+g_{V}\left(Y, D_{X}^{V} Z\right)+2 C_{V}\left(D_{X}^{V} V, Y, Z\right),
\end{aligned}
$$

where $C_{V}$ satisfies

$$
C_{V}(V, X, Y)=0
$$

Given two linearly independent vectors $V, W \in T_{x} M \backslash 0$, the flag curvature is defined by

$$
K(V, W):=\frac{g_{V}\left(R^{V}(V, W) W, V\right)}{g_{V}(V, V) g_{V}(W, W)-g_{V}(V, W)^{2}},
$$

where $R^{V}$ is the Chern curvature

$$
R^{V}(X, Y) Z=D_{X}^{V} D_{Y}^{V} Z-D_{Y}^{V} D_{X}^{V} Z-D_{[X, Y]}^{V} Z
$$

Then the Ricci curvature for $(M, F)$ is defined as

$$
\operatorname{Ric}(V)=\sum_{i=1}^{n-1} K\left(V, e_{i}\right)
$$

where $e_{1}, \ldots, e_{n-1}, \frac{V}{F(V)}$ form an orthonormal basis of $T_{x} M$ with respect to $g_{V}$.

For a given volume form $d \mu=\sigma(x) d x$ and a vector $y \in T_{x} M \backslash 0$, the distortion of $(M, F, d \mu)$ is defined by

$$
\tau(y):=\ln \frac{\sqrt{\operatorname{det}\left(g_{i j}(y)\right)}}{\sigma} .
$$

To measure the rate of changes of the distortion along geodesics, we define

$$
S(y):=\frac{d}{d t}[\tau(\dot{c}(t))]_{t=0},
$$

where $c(t)$ is the geodesic with $\dot{c}(0)=y$. $S$ is called the $S$-curvature.

Now we can introduce the weighted Ricci curvature on the Finsler manifolds, which was defined by Ohta in [6]. In the present paper, we reform it as follows.

Definition 2.1 [6] Let $(M, F, d \mu)$ be a Finsler $n$-manifold. Given a vector $V \in T_{x} M$, let $\gamma:(-\varepsilon, \varepsilon) \rightarrow M$ be a geodesic with $\gamma(0)=x, \dot{\gamma}(0)=V$. Define

$$
\dot{S}(V):=F^{-2}(V) \frac{\partial}{\partial t}[S(\gamma(t), \dot{\gamma}(t))]_{t=0},
$$


where $S(V)$ denotes the $S$-curvature at $(x, V)$. The weighted Ricci curvature of $(M, F, d \mu)$ is defined by

$$
\left\{\begin{array}{l}
\operatorname{Ric}_{n}(V):=\left\{\begin{array}{l}
\operatorname{Ric}(V)+\dot{S}(V), \\
-\infty, \quad \text { for } S(V)=0, \\
\text { otherwise, }
\end{array}\right. \\
\operatorname{Ric}_{N}(V):=\operatorname{Ric}(V)+\dot{S}(V)-\frac{S(V)^{2}}{(N-n) F(V)^{2}}, \quad \forall N \in(n, \infty), \\
\operatorname{Ric}_{\infty}(V):=\operatorname{Ric}(V)+\dot{S}(V) .
\end{array}\right.
$$

Here we will spend some words about the assumption of the nonpositive $S$-curvature in this paper. If the Finsler metric $F$ is reversible, then the $S$-curvature is homogeneous $S(-y)=-S(y)$ and hence $S \leq 0$ only if $S=0$. If $S=0$, then $\operatorname{Ric}_{N}=$ Ric for all $N$. For instance, the Busemann-Hausdorff measures on Berwald spaces satisfy $S=0$. Express a Rander metric $F=\alpha+\beta$ in terms of a Riemannian metric $h=\sqrt{h_{i j} y^{i} y^{j}}$ and a vector $W=W^{i} \frac{\partial}{\partial x^{i}}$ by

$$
F=\frac{\sqrt{\lambda h^{2}+W_{0}^{2}}}{\lambda}-\frac{W_{0}}{\lambda}, \quad W_{0}:=W_{i} y^{i}
$$

where $W_{i}:=h_{i j} W^{j}$ and $\lambda:=1-W_{i} W^{i}=1-h(x, W)^{2}$. Set

$$
h=|y|=\sqrt{\sum_{i=1}^{n}\left(y^{i}\right)^{2}}, \quad W=-2 c x+x Q+b,
$$

where $c<0$ is a constant, $Q=\left(q_{j}^{i}\right)$ is an anti-symmetric matrix and $b \in R^{n}$ is a constant vector. In [11], we know that $F$ has constant flag curvature $K=-c^{2}$ and $W_{0 ; 0}=-2 c h^{2}$. From [12], we further get $S=(n+1) c F<0$ by using the Busemann-Hausdorff measures. For more examples, we can refer to [13].

For a smooth function $u$ and a smooth vector field $V$ on $M$, we set $M_{u}:=\{x \in M \mid d u(x) \neq$ $0\}$ and $M_{V}:=\{x \in M \mid V(x) \neq 0\}$. If $V \neq 0$ on $M_{u}$, then the weighted gradient vector of $u$ on the weighted Riemannian manifold $\left(M, g_{V}\right)$ is defined by

$$
\nabla^{V} u:= \begin{cases}g^{i j}(V) \frac{\partial u}{\partial x^{j}} \frac{\partial}{\partial x^{i}} & \text { on } M_{V} \\ 0 & \text { on } M \backslash M_{V}\end{cases}
$$

The divergence of $V=V^{i} \frac{\partial}{\partial x^{i}}$ on $M$ with respect to an arbitrary volume form $d \mu=e^{\Phi} d x$ and the Finsler weighted Laplacian of $u$ on $\left(M, g_{V}\right)$ are defined by

$$
\operatorname{div} V:=\sum_{i=1}^{n}\left(\frac{\partial V^{i}}{\partial x^{i}}+V^{i} \frac{\partial \Phi}{\partial x^{i}}\right), \quad \Delta^{V} u:=\operatorname{div}\left(\nabla^{V} u\right),
$$

respectively.

Let $\mathcal{L}: T M \rightarrow T^{*} M$ be the Legendre transform. For a smooth function $u$ on $M$, the gradient vector and the Finsler-Laplacian of $u$ is defined by

$$
\nabla u:=\mathcal{L}^{-1}(d u), \quad \Delta u:=\operatorname{div}(\nabla u)
$$

In particular, on $M_{u}$ we have

$$
\nabla u=\nabla^{\nabla u} u, \quad \Delta u=\Delta^{\nabla u} u .
$$


Let $X=X^{i} \frac{\partial}{\partial x^{i}}$ be a differential vector field. Then the covariant derivative of $X$ by $v \in T_{x} M$ with reference vector $w \in T_{x} M \backslash 0$ is defined by

$$
D_{v}^{w} X(x):=\left\{v^{j} \frac{\partial X^{i}}{\partial x^{j}}(x)+\Gamma_{j k}^{i}(w) v^{j} X^{k}(x)\right\} \frac{\partial}{\partial x^{i}},
$$

where $\Gamma_{j k}^{i}$ denotes the coefficients of the Chern connection.

For a smooth vector field $V$ on $M$ and $x \in M_{V}$, we define $\nabla V(x) \in T_{x}^{*} M \otimes T_{x} M$ by using the covariant derivative as

$$
\nabla V(v):=D_{v}^{V} V(x) \in T_{x} M, \quad v \in T_{x} M
$$

We also set $\nabla^{2} u(x):=\nabla(\nabla u)(x)$ for the smooth function $u$ and $x \in M_{u}$. Then

$$
\nabla^{2} u(X, Y)=X Y(u)-D_{X}^{\nabla u} Y(u)=g_{\nabla u}\left(D_{X}^{\nabla u} \nabla u, Y\right), \quad \forall X,\left.Y \in T M\right|_{M_{u}}
$$

Let $\left\{e_{a}\right\}_{a=1}^{n}$ be a local orthonormal basis with respect to $g_{\nabla u}$ on $M_{u}$. Write $u_{a b}:=$ $g_{\nabla u}\left(\nabla^{2} u\left(e_{a}\right), e_{b}\right)$, then we have

$$
u_{a b}=u_{b a} .
$$

Let $(M, F)$ be a Finsler manifold. Define the distance function by

$$
d(p, q):=\inf _{\gamma} \int_{0}^{1} F(\gamma, \dot{\gamma}(t)) d t
$$

where the infimum is taken over all differentiable curves $\gamma:[0,1] \rightarrow M$ with $\gamma(0)=p$ and $\gamma(1)=q$.

Lemma 2.2 [3] Let $(M, F)$ be a Finsler n-manifold and $u: M \rightarrow R$ a smooth function. Then on $M_{u}$ we have

$$
\Delta u=\operatorname{tr}_{g_{\nabla u}}\left(\nabla^{2} u\right)-S(\nabla u)=\sum_{a} u_{a a}-S(\nabla u),
$$

where $u_{a a}=g_{\nabla u}\left(\nabla^{2} u\left(e_{a}\right), e_{a}\right)$ and $\left\{e_{a}\right\}_{a=1}^{n}$ is a local $g_{\nabla u}$-orthonormal basis on $M_{u}$.

Lemma 2.3 [1] Assume that $\operatorname{Ric}_{N} \geq 0$ for $N \in(n, \infty)$. Then the Laplacian of the distance function $\rho(x)=d(p, x)$ from any given point $p \in M$ can be estimated as follows:

$$
\Delta \rho \leq \frac{N-1}{\rho}
$$

in the sense of distributions on $M \backslash\{p\}$.

Lemma 2.4 [3] Let $(M, F)$ be a Finsler $n$-manifold and $\rho=d(p, \cdot)$ be the distance function from a fixed point $p$. Suppose that the flag curvature of $M$ satisfies $K \leq c$. Then for any vector $X$ on $M$, the following inequality holds whenever $\rho$ is smooth:

$$
\nabla^{2} \rho(X, X) \geq \operatorname{ct}_{c}(\rho)\left(g_{\nabla \rho}(X, X)-g_{\nabla \rho}(\nabla \rho, X)^{2}\right),
$$

where $\operatorname{ct}_{c}(\rho)$ is defined by (1.1). 


\section{Laplacian comparison theorems}

Theorem 3.1 Let $(M, F, d \mu)$ be a Finsler n-manifold with nonpositive flag curvature and nonpositive S-curvature. If the weighted Ricci curvature satisfies $\mathrm{Ric}_{n+1} \leq c$, then the following holds whenever the distance function $\rho$ is smooth:

$$
\Delta \rho \geq \operatorname{ct}_{c}(\rho)
$$

Proof Let $\rho(x)=d(p, x)$ be the distance function. If $\rho$ is smooth at $q \in M$, then it is also smooth near $q$. Let $S_{p}(\rho(q))$ be the forward geodesic sphere of radius $\rho(q)$ centered at $p$. Choosing the local $g_{\nabla \rho}$-orthonormal frame $E_{1}, \ldots, E_{n-1}$ of $S_{p}(\rho(q))$ near $q$, we get local vector fields $E_{1}, \ldots, E_{n-1}, E_{n}=\nabla \rho$ by parallel transport along geodesic rays. Using (2.1)(2.4), we have

$$
\begin{aligned}
\frac{d}{d \rho}\left[\nabla^{2} \rho\left(E_{i}, E_{j}\right)\right] & =\frac{d}{d \rho} g_{\nabla \rho}\left(D_{E_{i}}^{\nabla \rho} \nabla \rho, E_{j}\right)=g_{\nabla \rho}\left(D_{\nabla \rho}^{\nabla \rho} D_{E_{i}}^{\nabla \rho} \nabla \rho, E_{j}\right) \\
& =g_{\nabla \rho}\left(R^{\nabla \rho}\left(\nabla \rho, E_{i}\right) \nabla \rho, E_{j}\right)+g_{\nabla \rho}\left(D_{\left[\nabla \rho, E_{i}\right]}^{\nabla \rho} \nabla \rho, E_{j}\right) \\
& =-g_{\nabla \rho}\left(R^{\nabla \rho}\left(E_{i}, \nabla \rho\right) \nabla \rho, E_{j}\right)-g_{\nabla \rho}\left(D_{D_{E_{i}}^{\nabla \rho} \nabla \rho}^{\nabla \rho} \nabla \rho, E_{j}\right) \\
& =-g_{\nabla \rho}\left(R^{\nabla \rho}\left(E_{i}, \nabla \rho\right) \nabla \rho, E_{j}\right)-\sum_{k} g_{\nabla \rho}\left(D_{E_{i}}^{\nabla \rho} \nabla \rho, E_{k}\right) g_{\nabla \rho}\left(D_{E_{k}}^{\nabla \rho} \nabla \rho, E_{j}\right) .
\end{aligned}
$$

Consequently,

$$
\frac{d}{d \rho}\left(\operatorname{tr}_{\nabla \rho}\left(\nabla^{2} \rho\right)\right)=-\operatorname{Ric}(\nabla \rho)-\left\|\nabla^{2} \rho\right\|_{H S(\nabla \rho)}^{2} .
$$

Here $\|\cdot\|_{H S(\nabla \rho)}$ denotes the Hilbert-Schmidt norm with respect to $g_{\nabla \rho}$. We refer to [14] for details.

Since $M$ has nonpositive flag curvature, from Lemma 2.4 we see that the eigenvalues of $\nabla^{2} \rho$ are nonnegative. This yields

$$
\left\|\nabla^{2} \rho\right\|_{H S(\nabla \rho)}^{2} \leq\left(\operatorname{tr}_{\nabla \rho}\left(\nabla^{2} \rho\right)\right)^{2}
$$

Note that $\operatorname{Ric}_{N}=\operatorname{Ric}+\dot{S}-\frac{S^{2}}{N-n}$ and $\operatorname{Ric}_{N} \leq c$ for $N \geq n+1$, from (3.1) and (3.2) we have

$$
\begin{aligned}
\frac{d}{d \rho}\left(\operatorname{tr}_{\nabla \rho}\left(\nabla^{2} \rho\right)\right) & =-\operatorname{Ric}_{N}(\nabla \rho)+\dot{S}(\nabla \rho)-\frac{S(\nabla \rho)^{2}}{N-n}-\left\|\nabla^{2} \rho\right\|_{H S(\nabla \rho)}^{2} \\
& \geq-c+\dot{S}(\nabla \rho)-\frac{S(\nabla \rho)^{2}}{N-n}-\left(\operatorname{tr}_{\nabla \rho}\left(\nabla^{2} \rho\right)\right)^{2} .
\end{aligned}
$$

Notice that $S \leq 0$ and $\nabla^{2} \rho$ has nonnegative eigenvalues; from Lemma 2.2 we have

$$
\begin{aligned}
(\Delta \rho)^{2} & =\left(\operatorname{tr}_{\nabla \rho}\left(\nabla^{2} \rho\right)-S\right)^{2} \\
& =\left(\operatorname{tr}_{\nabla \rho}\left(\nabla^{2} \rho\right)\right)^{2}+S^{2}-2 S \operatorname{tr}_{\nabla \rho}\left(\nabla^{2} \rho\right) \\
& \geq\left(\operatorname{tr}_{\nabla \rho}\left(\nabla^{2} \rho\right)\right)^{2}+S^{2} \\
& \geq\left(\operatorname{tr}_{\nabla \rho}\left(\nabla^{2} \rho\right)\right)^{2}+\frac{S^{2}}{N-n}
\end{aligned}
$$


for $N-n \geq 1$. On the other hand, it is easy to see that $\frac{d}{d \rho} S=\dot{S}$ since $F(\nabla \rho)=1$. Combining (3.3) and (3.4), and using Lemma 2.2 again, we obtain

$$
\frac{d}{d \rho}(\Delta \rho) \geq-(\Delta \rho)^{2}-c
$$

By a simple argument, (3.5) can be rewritten as

$$
\frac{d}{d \rho}\left(\Delta \rho-\operatorname{ct}_{c}(\rho)\right) \geq\left(\operatorname{ct}_{c}(\rho)\right)^{2}-(\Delta \rho)^{2}
$$

Set $A=\Delta \rho-\operatorname{ct}_{c}(\rho), B=\Delta \rho+\operatorname{ct}_{c}(\rho)$, then (3.6) becomes

$$
\frac{d A}{d \rho}+A B \geq 0
$$

Since $M$ has nonpositive flag curvature and nonpositive $S$-curvature, from Lemma 2.4 we get

$$
\Delta \rho \geq \Delta \rho+S=\operatorname{tr}_{\nabla \rho}\left(\nabla^{2} \rho\right) \geq \frac{n-1}{\rho}
$$

which implies that there exists $\varepsilon>0$ such that

$$
A(\rho) \geq \frac{n-1}{\rho}-\operatorname{ct}_{c}(\rho) \geq 0, \quad \forall \rho \in(0, \varepsilon] .
$$

From (3.7) we have

$$
\frac{d}{d \rho}\left(A(\rho) \exp \left(\int_{\varepsilon}^{\rho} B(t) d t\right)\right) \geq 0,
$$

which yields $A(\rho) \geq 0$, i.e., $\Delta \rho \geq \operatorname{ct}_{c}(\rho)$.

If $M$ has nonpositive $S$-curvature, then

$$
\Delta \rho=\operatorname{tr}_{\nabla \rho}\left(\nabla^{2} \rho\right)-S \geq \operatorname{tr}_{\nabla \rho}\left(\nabla^{2} \rho\right)
$$

Thus from Lemma 2.4, we get the following.

Proposition 3.2 Let $(M, F, d \mu)$ be a Finsler n-manifold with nonpositive S-curvature. If the flag curvature satisfies $K \leq c$, then the following holds whenever the distance function $\rho$ is smooth:

$$
\Delta \rho \geq(n-1) \operatorname{ct}_{c}(\rho)
$$

\section{Volume comparison theorems}

Let $(M, F, d \mu)$ be a Finsler $n$-manifold. For a fixed point $p \in M$, define

$$
I_{p}:=\left\{v \in T_{p} M \mid F(v)=1\right\}, \quad c(v):=\sup \left\{t>0 \mid d_{F}(p, \exp (t v))=t\right\},
$$




$$
\begin{array}{ll}
\mathbf{C}(p):=\left\{c(v) v \mid c(v)<\infty, v \in I_{p}\right\}, & C(p):=\exp \mathbf{C}(p), \quad i_{p}:=\inf \left\{c(v) \mid v \in I_{p}\right\} \\
\mathbf{D}(p):=\left\{t v \mid 0 \leq t<c(v), v \in I_{p}\right\}, & D(p):=\exp \mathbf{D}(p) .
\end{array}
$$

Then $D(p)=M \backslash C(p)$. Let $\left\{\theta^{\alpha} \mid \alpha=1, \ldots, n-1\right\}$ be the local coordinates that are intrinsic to $I_{p}$. For any $q \in D(p)$, the polar coordinates of $q$ are defined by $(\rho, \theta)=$ $\left(\rho(q), \theta^{1}(q), \ldots, \theta^{n-1}(q)\right)$, where $\rho(q)=F(v), \theta^{\alpha}(q)=\theta^{\alpha}\left(\frac{v}{F(v)}\right)$ and $v=\exp _{p}^{-1}(q)$. Since $\frac{\partial}{\partial \rho}=$ $\nabla \rho$, we conclude

$$
g_{\nabla \rho}\left(\frac{\partial}{\partial \rho}, \frac{\partial}{\partial \theta^{\alpha}}\right)=0, \quad \forall \alpha, \quad g_{\nabla \rho}\left(\frac{\partial}{\partial \rho}, \frac{\partial}{\partial \rho}\right)=1
$$

in view of the Gauss lemma. Therefore, if $d \mu=\sigma(\rho, \theta) d \rho \wedge d \theta$, then from the definition of the Finsler-Laplacian of a function we have

$$
\Delta \rho=\frac{\partial}{\partial \rho} \log \sigma
$$

Proposition 4.1 Let $(M, F, d \mu)$ be a complete Finsler n-manifold with nonpositive Scurvature. If the flag curvature satisfies $K \leq c$, then the function

$$
\frac{\operatorname{vol}_{F}^{d \mu}\left(B_{p}^{+}(\rho)\right)}{\operatorname{vol}\left(\mathbf{B}_{c}^{n}(\rho)\right)}
$$

is monotone increasing for $0<\rho<i_{p}$, where $i_{p}$ is the injectivity radius of $p$ and $\mathbf{B}_{c}^{n}(\rho)$ denotes the geodesic ball of radius $\rho$ in a space form of constant sectional curvature c. In particular, for the Busemann-Hausdorff volume form $d \mu=\sigma_{B H} d x$, one has

$$
\operatorname{vol}_{F}^{d \mu}\left(B_{p}^{+}(\rho)\right) \geq \operatorname{vol}\left(\mathbf{B}_{c}^{n}(\rho)\right), \quad \rho \leq i_{p}
$$

Proof By (4.1), Proposition 3.2 and the assumption of Proposition 4.1, we have

$$
\frac{\partial}{\partial \rho} \log \sigma \geq(n-1) \operatorname{ct}_{c}(\rho)=\frac{d}{d \rho} \log \left(s_{c}(\rho)^{n-1}\right),
$$

which implies that the function

$$
\frac{\sigma(\rho, \theta)}{\mathbf{s}_{c}(\rho)^{n-1}}
$$

is monotone increasing with respect to $\rho$, where

$$
\mathrm{S}_{c}(\rho):= \begin{cases}\frac{\sin (\sqrt{c} \rho)}{\sqrt{c}}, & c>0 ; \\ \rho, & c=0 ; \\ \frac{\sinh (\sqrt{-c} \rho)}{\sqrt{-c}}, & c<0 .\end{cases}
$$

Let $\mathbf{D}_{p}(\rho):=\left\{v \in I_{p} \mid \rho v \in \mathbf{D}_{p}\right\}$. It is easy to see that $\mathbf{D}_{p}(\rho)=I_{p}$ for $\rho<i_{p}$. Set

$$
\sigma_{p}(\rho):=\int_{\mathbf{D}_{p}(\rho)} \sigma(\rho, \theta) d \theta, \quad \sigma_{c, n}(\rho):=\operatorname{vol}\left(\mathbf{S}^{n-1}(1)\right) \mathrm{s}_{c}(\rho)^{n-1} .
$$


Then for $\rho<i_{p}$,

$$
\operatorname{vol}_{F}^{d \mu}\left(B_{p}^{+}(\rho)\right)=\int_{0}^{\rho} \sigma_{p}(\rho) d \rho, \quad \operatorname{vol}\left(\mathbf{B}_{c}^{n}(\rho)\right)=\int_{0}^{\rho} \sigma_{c, n}(\rho) d \rho .
$$

For two positive integrable functions $f$ and $g$, if $\frac{f}{g}$ is monotone increasing, then the function

$$
\frac{\int_{0}^{r} f(t) d t}{\int_{0}^{r} g(t) d t}
$$

is also monotone increasing (see Lemma 5.1 in [4] for details). From this statement, one finds that $\frac{\sigma_{p}(\rho)}{\sigma_{c, n}(\rho)}$ is monotone increasing, and also the function

$$
\frac{\int_{0}^{\rho} \int_{\mathbf{D}_{p}(\rho)} \sigma(\rho, \theta) d \theta d \rho}{\operatorname{vol}\left(\mathbf{S}^{n-1}(1)\right) \int_{0}^{\rho} \mathbf{s}_{c}(\rho)^{n-1} d \rho}=\frac{\operatorname{vol}_{F}^{d \mu}\left(B_{p}^{+}(\rho)\right)}{\operatorname{vol}\left(\mathbf{B}_{c}^{n}(\rho)\right)}
$$

is monotone increasing for $\rho<i_{p}$.

To prove (4.2), we only need to show

$$
\lim _{\rho \rightarrow 0} \frac{\operatorname{vol}_{F}^{d \mu}\left(B_{p}^{+}(\rho)\right)}{\operatorname{vol}\left(\mathbf{B}_{c}^{n}(\rho)\right)}=1
$$

when $d \mu=\sigma_{B H} d x$. Since

$$
\lim _{\rho \rightarrow 0} \frac{\operatorname{vol}\left(\mathbf{B}_{c}^{n}(\rho)\right)}{\operatorname{vol}\left(\mathbf{B}_{0}^{n}(\rho)\right)}=\lim _{\rho \rightarrow 0} \frac{\int_{0}^{\rho} \mathbf{s}_{c}(t)^{n-1} d t}{\int_{0}^{\rho} t^{n-1} d t}=1
$$

it is sufficient to prove

$$
\lim _{\rho \rightarrow 0} \frac{\operatorname{vol}_{F}^{d \mu}\left(B_{p}^{+}(\rho)\right)}{\operatorname{vol}\left(\mathbf{B}_{0}^{n}(\rho)\right)}=1,
$$

which can be directly obtained from [2].

By using Theorem 3.1, we can get the following result similarly.

Proposition 4.2 Let $(M, F, d \mu)$ be a complete and simply connected Finsler n-manifold with nonpositive flag curvature and nonpositive S-curvature. If the weighted Ricci curvature satisfies $\operatorname{Ric}_{n+1} \leq c<0$, then the function

$$
\frac{\operatorname{vol}_{F}^{d \mu}\left(B_{p}^{+}(\rho)\right)}{\operatorname{vol}\left(\mathbf{B}_{c}^{2}(\rho)\right)}
$$

is monotone increasing. In particular, for the Busemann-Hausdorff volume form $d \mu=$ $\sigma_{B H} d x$, one has

$$
\operatorname{vol}_{F}^{d \mu}\left(B_{p}^{+}(\rho)\right) \geq \frac{\operatorname{vol}_{F}^{d \mu}\left(B_{p}^{+}(1)\right)}{\operatorname{vol}_{F}^{d \mu}\left(\mathbf{B}_{c}^{2}(1)\right)} \operatorname{vol}\left(\mathbf{B}_{c}^{2}(\rho)\right), \quad \forall \rho \geq 1 .
$$


Define reversibility $\lambda=\lambda(M, F)$ as follows:

$$
\lambda:=\sup _{X \in T M \backslash 0} \frac{F(-X)}{F(X)} .
$$

Obviously, $\lambda \in[1, \infty]$, and $\lambda=1$ if and only if $(M, F)$ is reversible.

In what follows, we shall generalize Calabi-Yau's linear volume growth theorem to Finsler manifolds with an arbitrary volume form.

Theorem 4.3 Let $(M, F, d \mu)$ be a complete noncompact Finsler n-manifold with finite reversibility $\lambda$. If the weighted Ricci curvature satisfies $\operatorname{Ric}_{N} \geq 0, N \in(n, \infty)$, then

$$
\begin{aligned}
& \operatorname{vol}_{F}^{d \mu}\left(B_{p}^{+}(R)\right) \geq C\left(N, \lambda, \operatorname{vol}_{F}^{d \mu}\left(B_{p}^{+}(1)\right)\right) R, \\
& \operatorname{vol}_{F}^{d \mu}\left(B_{p}^{-}(R)\right) \geq C\left(N, \lambda, \operatorname{vol}_{F}^{d \mu}\left(B_{p}^{-}(1)\right)\right) R,
\end{aligned}
$$

where $B_{p}^{+}(R)$ (resp. $B_{p}^{-}(R)$ ) denotes the forward (resp. backward) geodesic ball of radius $R$ centered at $p$ and $C$ denotes the constant depending on $N, \lambda$, and $\operatorname{vol}_{F}^{d \mu}\left(B_{p}^{+}(1)\right)($ resp. $\left.\operatorname{vol}_{F}^{d \mu}\left(B_{p}^{-}(1)\right)\right)$.

Proof Let $x_{0} \in \partial B_{p}^{-}(R)$ be a given point. Namely, $d\left(x_{0}, p\right)=R$. Let $\rho$ be the distance function $\rho(x)=d\left(x_{0}, x\right)$. Then $F(\nabla \rho):=\|\nabla \rho\|=1$. From Lemma 2.3 we have

$$
\Delta \rho \leq \frac{N-1}{\rho}
$$

which yields

$$
\Delta^{\nabla \rho} \rho^{2}=2 \rho \Delta \rho+2\|\nabla \rho\|^{2} \leq 2(N-1)+2=2 N .
$$

Therefore, for any nonnegative function $\varphi \in C_{0}^{\infty}(M)$, one obtains

$$
\int_{M} \varphi \Delta^{\nabla \rho} \rho^{2} d \mu \leq 2 N \int_{M} \varphi d \mu
$$

Set

$$
\psi(t):= \begin{cases}1, & 0 \leq t \leq R-\lambda \\ \frac{R+1-t}{1+\lambda}, & R-\lambda \leq t \leq R+1 \\ 0, & t \geq R+1\end{cases}
$$

for any $R>\lambda$. If $\varphi(x)=\psi(\rho(x))$, then $\varphi(x)$ is a Lipschitz continuous function and supp $\varphi \subset$ $B_{x_{0}}^{+}(R+1)$. Since the Stokes formula still holds for Lipschitz continuous functions, we have

$$
\begin{aligned}
\int_{M} \varphi \Delta^{\nabla \rho} \rho^{2} d \mu & =-\int_{B_{x_{0}}^{+}(R+1)} g_{\nabla \rho}\left(\nabla^{\nabla \rho} \varphi, \nabla^{\nabla \rho} \rho^{2}\right) d \mu \\
& =-2 \int_{B_{x_{0}}^{+}(R+1)} \psi^{\prime}(\rho(x)) \rho\|\nabla \rho\|^{2} d \mu
\end{aligned}
$$




$$
\begin{aligned}
& =\frac{2}{1+\lambda} \int_{B_{x_{0}}^{+}(R+1) \backslash B_{x_{0}}^{+}(R-\lambda)} \rho d \mu \\
& \geq \frac{2(R-\lambda)}{1+\lambda} \operatorname{vol}_{F}^{d \mu}\left(B_{x_{0}}^{+}(R+1) \backslash B_{x_{0}}^{+}(R-\lambda)\right) .
\end{aligned}
$$

It follows from (4.4) that

$$
\begin{aligned}
& \frac{2(R-\lambda)}{1+\lambda} \operatorname{vol}_{F}^{d \mu}\left(B_{x_{0}}^{+}(R+1) \backslash B_{x_{0}}^{+}(R-\lambda)\right) \\
& \quad \leq 2 N \int_{M} \varphi d \mu=2 N \int_{B_{x_{0}}^{+}(R+1)} \varphi d \mu \\
& \quad \leq 2 N \int_{B_{x_{0}}^{+}(R+1)} d \mu=2 N \operatorname{vol}_{F}^{d \mu}\left(B_{x_{0}}^{+}(R+1)\right) .
\end{aligned}
$$

Notice that $d(p, q) \leq \lambda d(q, p), \forall p, q \in M$, it is easy to find from the triangle inequality that

$$
B_{p}^{+}(1) \subset B_{x_{0}}^{+}(R+1) \backslash B_{x_{0}}^{+}(R-\lambda), \quad \forall R>\lambda .
$$

Therefore, from (4.7) and (4.8) we have

$$
\begin{aligned}
2 N \operatorname{vol}_{F}^{d \mu}\left(B_{x_{0}}^{+}(R+1)\right) & \geq \frac{2(R-\lambda)}{1+\lambda} \operatorname{vol}_{F}^{d \mu}\left(B_{x_{0}}^{+}(R+1) \backslash B_{x_{0}}^{+}(R-\lambda)\right) \\
& \geq \frac{2(R-\lambda)}{1+\lambda} \operatorname{vol}_{F}^{d \mu} B_{p}^{+}(1) .
\end{aligned}
$$

On the other hand, it is not hard to see that $B_{x_{0}}^{+}(R+1) \subset B_{p}^{+}((\lambda+1)(R+1))$. Combining this and (4.9) one obtains

$$
\operatorname{vol}_{F}^{d \mu}\left(B_{p}^{+}((\lambda+1)(R+1))\right) \geq \frac{(R-\lambda)}{N(1+\lambda)} \operatorname{vol}_{F}^{d \mu} B_{p}^{+}(1) .
$$

Replacing $(\lambda+1)(R+1)$ by $R$, we have

$$
\begin{aligned}
\operatorname{vol}_{F}^{d \mu}\left(B_{p}^{+}(R)\right) & \geq \frac{\left(\frac{R}{\lambda+1}-(1+\lambda)\right)}{N(1+\lambda)} \operatorname{vol}_{F}^{d \mu} B_{p}^{+}(1) \\
& \geq C\left(N, \lambda, \operatorname{vol}_{F}^{d \mu}\left(B_{p}^{+}(1)\right)\right) R
\end{aligned}
$$

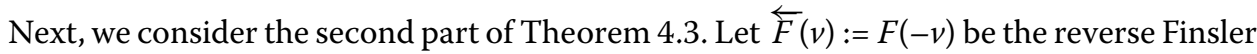
metric of $F$. If $F$ reversible, then $\overleftarrow{F}=F$. We put an arrow $\leftarrow$ on those quantities associated with $\overleftarrow{F}$. For example,

$$
\overleftarrow{d}(x, y)=d(y, x), \quad \overleftarrow{\nabla} u=-\nabla(-u), \quad \overleftarrow{\Delta u}=-\Delta(-u), \quad \overleftarrow{\operatorname{Ric}}_{N}(v)=\operatorname{Ric}_{N}(-v)
$$

If the weighted Ricci curvature of $F$ satisfies $\operatorname{Ric}_{N} \geq 0$, then for the reverse Finsler metric $\overleftarrow{F}, \overleftarrow{\mathrm{Ric}}_{N} \geq 0$. Moreover, the corresponding Laplacian comparison theorem still holds [6]. Since the curvature condition is common between $F$ and $\overleftarrow{F}$, the assertion for $B^{-}$w.r.t. $F$ follows from that for $B^{+}$w.r.t. $\overleftarrow{F}$. So the proof is omitted here. 
Corollary 4.4 A complete noncompact Finsler n-manifold with nonnegative weighted Ricci curvature and finite reversibility must have infinite volume.

Theorem 4.5 Let $(M, F, d \mu)$ be a complete and simply connected Finsler n-manifold, with nonpositive flag curvature and nonpositive S-curvature. If the weighted Ricci curvature satisfies $\operatorname{Ric}_{n+1} \leq-(n-1) b^{2}(b>0)$, then for any fixed $\varepsilon>0$, there exists a positive constant $c(n, b, \varepsilon)$ such that when $\rho \geq \varepsilon$, one has

$$
\operatorname{vol}_{F}^{d \mu}\left(B_{p}^{+}(\rho)\right) \geq c(n, b, \varepsilon) e^{b \rho},
$$

where $\operatorname{vol}_{F}^{d \mu}\left(B_{p}^{+}(\rho)\right)$ is the volume of the forward geodesic ball centered at $p \in M$ with radius $\rho$.

Proof Using (3.1), Lemma 2.2, and Definition 2.1, we have

$$
\frac{d}{d \rho}(\Delta \rho)=-\operatorname{Ric}_{\infty}(\nabla \rho)-\left\|\nabla^{2} \rho\right\|_{H S(\nabla \rho)}^{2} .
$$

Combining (4.1) one gets

$$
\frac{\partial^{2} \sigma(\rho, \theta)}{\partial \rho^{2}}=\sigma(\Delta \rho)^{2}-\sigma \operatorname{Ric}_{\infty}(\nabla \rho)-\sigma\left\|\nabla^{2} \rho\right\|_{H S(\nabla \rho)}^{2},
$$

which together with (3.2) and (3.4) yields

$$
\frac{\partial^{2} \sigma}{\partial \rho^{2}} \geq-\sigma \operatorname{Ric}_{N}(\nabla \rho)
$$

for $N \in[n+1, \infty]$.

On the other hand, for a Riemannian manifold $(\bar{M}, \bar{g})$ with constant sectional curvature $-b^{2}$, we have $\bar{\sigma}=\sqrt{\bar{g}}$ and

$$
\begin{aligned}
& (\bar{\Delta} \rho)^{2}=(n-1)\left\|\bar{\nabla}^{2} \rho\right\|^{2}, \\
& \frac{\partial^{2} \bar{\sigma}}{\partial \rho^{2}}=-\bar{\sigma} \overline{\operatorname{Ric}}(\bar{\nabla} \rho)+\frac{n-2}{n-1} \frac{\bar{\sigma}^{\prime 2}}{\bar{\sigma}} \quad\left(\bar{\sigma}^{\prime}:=\frac{\partial \bar{\sigma}}{\partial \rho}\right) .
\end{aligned}
$$

Set $\Omega=: \sigma \bar{\sigma}^{-\frac{1}{n-1}}$. Then

$$
\Omega^{\prime}=\Omega\left(\frac{\sigma^{\prime}}{\sigma}-\frac{1}{n-1} \frac{\bar{\sigma}^{\prime}}{\bar{\sigma}}\right)=\Omega\left(\Delta \rho-\frac{1}{n-1} \bar{\Delta} \rho\right) .
$$

By (4.11) and (4.13), and the assumption of Theorem 4.5, we have

$$
\begin{aligned}
\Omega^{\prime \prime}= & \Omega\left(\frac{\sigma^{\prime}}{\sigma}-\frac{1}{n-1} \frac{\bar{\sigma}^{\prime}}{\bar{\sigma}}\right)^{2}+\Omega\left[\frac{\sigma^{\prime \prime}}{\sigma}-\left(\frac{\sigma^{\prime}}{\sigma}\right)^{2}-\frac{1}{n-1} \frac{\bar{\sigma}^{\prime \prime}}{\bar{\sigma}}+\frac{1}{n-1}\left(\frac{\bar{\sigma}^{\prime}}{\bar{\sigma}}\right)^{2}\right] \\
\geq & \Omega\left(\frac{\sigma^{\prime}}{\sigma}-\frac{1}{n-1} \frac{\bar{\sigma}^{\prime}}{\bar{\sigma}}\right)^{2} \\
& +\Omega\left[-\operatorname{Ric}_{N}(\nabla \rho)-\left(\frac{\sigma^{\prime}}{\sigma}\right)^{2}+\frac{1}{n-1} \overline{\operatorname{Ric}}(\bar{\nabla} \rho)+\frac{1}{(n-1)^{2}}\left(\frac{\bar{\sigma}^{\prime}}{\bar{\sigma}}\right)^{2}\right]
\end{aligned}
$$




$$
\begin{aligned}
& \geq-\frac{2}{n-1} \frac{\bar{\sigma}^{\prime}}{\bar{\sigma}} \Omega\left(\frac{\sigma^{\prime}}{\sigma}-\frac{1}{n-1} \frac{\bar{\sigma}^{\prime}}{\bar{\sigma}}\right) \\
& =-\frac{2}{n-1} \frac{\bar{\sigma}^{\prime}}{\bar{\sigma}} \Omega^{\prime},
\end{aligned}
$$

which implies

$$
\left(\bar{\sigma} \frac{2}{n-1} \Omega^{\prime}\right)^{\prime}=\bar{\sigma} \frac{2}{n-1}\left(\Omega^{\prime \prime}+\frac{2}{n-1} \frac{\bar{\sigma}^{\prime}}{\bar{\sigma}} \Omega^{\prime}\right) \geq 0 .
$$

Therefore $\bar{\sigma} \frac{2}{n-1} \Omega^{\prime}$ is increasing in $\rho$. Hence when $\rho \geq \varepsilon$,

$$
\begin{aligned}
\Omega^{\prime}(\rho) & \geq \bar{\sigma}^{-\frac{2}{n-1}}(\rho) \bar{\sigma}^{\frac{2}{n-1}}(\varepsilon) \Omega^{\prime}(\varepsilon) \\
& \geq \lim _{\varepsilon \rightarrow 0} \bar{\sigma}^{-\frac{2}{n-1}}(\rho) \bar{\sigma}^{\frac{2}{n-1}}(\varepsilon) \sigma(\varepsilon) \bar{\sigma}^{-\frac{1}{n-1}}(\varepsilon)\left(\frac{\sigma^{\prime}}{\sigma}(\varepsilon)-\frac{1}{n-1} \frac{\bar{\sigma}^{\prime}}{\bar{\sigma}}(\varepsilon)\right) .
\end{aligned}
$$

Notice that $\sigma(\varepsilon, \theta) \sim \varepsilon^{n-1}, \bar{\sigma}(\varepsilon, \theta) \sim \varepsilon^{n-1}(\varepsilon \rightarrow 0)$. We obtain $\Omega^{\prime}(\rho) \geq 0$, which means that $\Omega=\sigma \bar{\sigma}^{-\frac{1}{n-1}}$ is also increasing in $\rho$. It is well known that

$$
\bar{\sigma}(\rho)=\left(\frac{\sinh (b \rho)}{b}\right)^{n-1} .
$$

Thus when $\rho \geq \varepsilon$, one has

$$
\frac{b \sigma(\rho, \theta)}{\sinh (b \rho)} \geq \frac{b \sigma(\varepsilon, \theta)}{\sinh (b \varepsilon)} \sim \varepsilon^{n-2}
$$

as $\varepsilon \rightarrow 0$, which shows that there exists $c=c(n, b)$ such that

$$
\sigma(\rho, \theta) \geq c \varepsilon^{n-2} \sinh (b \rho) .
$$

Consequently,

$$
\begin{aligned}
\operatorname{vol}_{F}^{d \mu}\left(B_{p}^{+}(\rho)\right) & =\int_{0}^{\rho} d t \int_{\mathbf{D}_{p}(\rho)} \sigma(t, \theta) d \theta \\
& \geq c \omega^{n-1} \varepsilon^{n-2} \int_{0}^{\rho} \sinh (b t) d t \\
& \geq c(n, b, \varepsilon) e^{b \rho},
\end{aligned}
$$

where $\omega^{n-1}$ denotes the volume of the unit sphere $\mathbf{S}^{n-1}$.

By similar argument, we also have the following result.

Proposition 4.6 Let $(M, F, d \mu)$ be a complete and simply connected Finsler n-manifold with nonpositive $S$-curvature. If the flag curvature satisfies $K \leq-b^{2}<0$, then the volume of the forward geodesic ball of $M$ grows at least exponentially.

Remark 4.7 Theorem 4.5 and Proposition 4.6 can also be deduced from Proposition 4.1 and Proposition 4.2. 
In [15], Milnor proved that the fundamental group of a compact Riemannian manifold of negative sectional curvature has exponential growth. Then this result was generalized to the case of negative Ricci curvature and nonpositive sectional curvature in [16] and [17]. The key point of the proof is to give a lower bound estimate for the volume of the geodesic balls of the universal covering space. In [3] and [4], the results were also generalized to the Finsler setting. By using the same method, we get another version of Milnor's results in Finsler geometry.

Theorem 4.8 Let $(M, F, d \mu)$ be a compact Finsler n-manifold with nonpositive S-curvature. Suppose that one of the following two conditions holds:

(i) the flag curvature satisfies $K \leq-b^{2}<0$;

(ii) $M$ has nonpositive flag curvature and $\operatorname{Ric}_{n+1} \leq-(n-1) b^{2}<0$.

Then the fundamental group of $M$ grows at least exponentially.

\section{Mckean type eigenvalue estimates}

Let $(M, F, d \mu)$ be a Finsler manifold, $\Omega \subset M$ be a domain with compact closure and nonempty boundary $\partial \Omega$. The first eigenvalue $\lambda_{1}(\Omega)$ is defined by

$$
\lambda_{1}(\Omega)=\inf _{u \in W_{0}^{1,2}(\Omega) \backslash\{0\}}\left\{\frac{\int_{\Omega}\left(F^{*}(d u)\right)^{2} d \mu}{\int_{\Omega} u^{2} d \mu}\right\},
$$

where $W_{0}^{1,2}(\Omega)$ is the completion of $C_{0}^{\infty}(\Omega)$. If $\Omega_{1} \subset \Omega_{2}$ are bounded domains, then $\lambda_{1}\left(\Omega_{1}\right) \geq \lambda_{1}\left(\Omega_{2}\right) \geq 0$. Thus, if $\Omega_{1} \subset \Omega_{2} \subset \cdots \subset M$ are bounded domains such that $\bigcup \Omega_{i}=$ $M$, then the limit

$$
\lambda_{1}(M)=\lim _{i \rightarrow \infty} \lambda_{1, p}\left(\Omega_{i}\right) \geq 0
$$

exists, and it is independent of the choice of $\left\{\Omega_{i}\right\}$.

Theorem 5.1 Let $(M, F, d \mu)$ be a complete noncompact and simply connected Finsler nmanifold with finite reversibility $\lambda$ and nonpositive $S$-curvature. If the flag curvature satisfies $K \leq-a^{2}(a>0)$, then

$$
\lambda_{1}(M) \geq \frac{(n-1)^{2} a^{2}}{4 \lambda^{2}} .
$$

Proof For $R>\delta>0$, set $\Omega_{\delta}=B_{p}(R) \backslash B_{p}(\delta)$, where $B_{p}(R)$ denotes the forward geodesic ball of radius $R$ centered at $p$. Then $\rho(x)=d(p, x)$ is differentiable in $\Omega_{\delta}$ and $\nabla \rho$ is a smooth vector field in $\Omega_{\delta}$. Let $f \in C_{0}^{\infty}\left(\Omega_{\delta}\right)$. Notice that $F(\nabla \rho)=1$, and we have

$$
\begin{aligned}
\operatorname{div}\left(f^{2} \nabla \rho\right) & =f^{2} \Delta \rho+2 f \nabla \rho(f) \\
& \geq f^{2} \Delta \rho-2 \lambda|f| F^{*}(d f) F(\nabla \rho) \\
& =f^{2} \Delta \rho-2 \lambda|f| F^{*}(d f),
\end{aligned}
$$


where $F^{*}$ is the dual Finsler metric of $F$. Since $K \leq-a^{2}$, it is known from Proposition 3.2 that $\Delta \rho \geq(n-1) a \operatorname{coth}(a \rho)$. Hence

$$
\begin{aligned}
\operatorname{div}\left(f^{2} \nabla \rho\right) & \geq f^{2}(n-1) a \operatorname{coth}(a \rho)-2 \lambda|f| F^{*}(d f) \\
& \geq f^{2}(n-1) a \operatorname{coth}(a R)-\lambda\left(\varepsilon f^{2}+\frac{1}{\varepsilon} F^{*}(d f)^{2}\right)
\end{aligned}
$$

holds for any $\varepsilon>0$. Integrating both sides of (5.1) over $\Omega_{\delta}$ and using the divergence theorem, we obtain

$$
\begin{aligned}
0= & \int_{\Omega_{\delta}} \operatorname{div}\left(f^{2} \nabla \rho\right) d \mu \geq(n-1) a \operatorname{coth}(a R) \int_{\Omega_{\delta}} f^{2} d \mu \\
& -\lambda \int_{\Omega_{\delta}}\left(\varepsilon f^{2}+\frac{1}{\varepsilon} F^{*}(d f)^{2}\right) d \mu .
\end{aligned}
$$

Therefore,

$$
\int_{\Omega_{\delta}} F^{*}(d f)^{2} d \mu \geq \frac{\varepsilon}{\lambda}((n-1) a \operatorname{coth}(a R)-\varepsilon \lambda) \int_{\Omega_{\delta}} f^{2} d \mu .
$$

Choosing $\varepsilon=\frac{(n-1) a \operatorname{coth}(a R)}{2 \lambda}$, one has

$$
\int_{\Omega_{\delta}} F^{*}(d f)^{2} d \mu \geq\left[\frac{(n-1) a \operatorname{coth}(a R)}{2 \lambda}\right]^{2} \int_{\Omega_{\delta}} f^{2} d \mu
$$

Letting $\delta \rightarrow 0$, we get

$$
\int_{B_{p}(R)} F^{*}(d f)^{2} d \mu \geq\left[\frac{(n-1) a \operatorname{coth}(a R)}{2 \lambda}\right]^{2} \int_{B_{p}(R)} f^{2} d \mu .
$$

Since $f$ is arbitrary, the formula above means

$$
\lambda_{1}\left(B_{p}(R)\right) \geq\left[\frac{(n-1) a \operatorname{coth}(a R)}{2 \lambda}\right]^{2} .
$$

Note that $(M, F, d \mu)$ is a complete noncompact and simply connected Finsler manifold. Letting $R \rightarrow \infty$, we have

$$
\lambda_{1}(M) \geq\left[\frac{(n-1) a}{2 \lambda}\right]^{2} .
$$

By using Theorem 3.1 and a similar argument, we can also prove the following result.

Theorem 5.2 Let $(M, F, d \mu)$ be a complete noncompact and simply connected Finsler nmanifold with finite reversibility $\lambda$, nonpositive flag curvature and nonpositive $S$-curvature. If the weighted Ricci curvature satisfies $\operatorname{Ric}_{n+1} \leq-a^{2}(a>0)$, then

$$
\lambda_{1}(M) \geq \frac{a^{2}}{4 \lambda^{2}} .
$$


Competing interests

The authors declare that they have no competing interests.

\section{Authors' contributions}

All authors read and approved the final manuscript.

\section{Author details}

${ }^{1}$ Department of Mathematics, Tongji University, Siping Road, Shanghai, China. ${ }^{2}$ Department of Mathematics and Computer Science, Tongling University, Beijing Road, Tongling, 244000, China.

\section{Acknowledgements}

This work is supported partly by NNSFC (no. 10971239, 11171253) and NSFHE (KJ2012B197). The authors would like to thank the referees for their useful comments on the manuscript.

Received: 1 December 2013 Accepted: 20 February 2014 Published: 04 Mar 2014

\section{References}

1. Ohta, S, Sturm, KT: Heat flow on Finsler manifolds. Commun. Pure Appl. Math. 62, 1386-1433 (2009)

2. Shen, ZM: Volume comparison and its applications in Riemann-Finsler geometry. Adv. Math. 128, 306-328 (1997)

3. Wu, BY, Xin, YL: Comparison theorems in Finsler geometry and their applications. Math. Ann. 337, 177-196 (2007)

4. Wu, BY: Volume form and its applications in Finsler geometry. Publ. Math. (Debr.) 78(3-4), 723-741 (2011)

5. Zhao, W, Shen, YB: A universal volume comparison theorem for Finsler manifolds and related results. Can. J. Math. 65, 1401-1435 (2013)

6. Ohta, S: Finsler interpolation inequalities. Calc. Var. Partial Differ. Equ. 36, 211-249 (2009)

7. Ding, Q: A new Laplacian comparison theorem and the estimate of eigenvalues. Chin. Ann. Math., Ser. B 15, 35-42 (1994)

8. Calabi, E: On manifolds with nonnegative Ricci curvature II. Not. Am. Math. Soc. 22, A-205 (1975). Abstract No. $720-53-6$

9. Yau, ST: Some function-theoretic properties of complete Riemannian manifold and their applications to geometry. Indiana Univ. Math. J. 25, 659-670 (1976)

10. Mckean, HP: An upper bound for spectrum of $\Delta$ on a manifold of negative curvature. J. Differ. Geom. 4, 359-366 (1970)

11. Bao, DW, Robles, C, Shen, ZM: Zermelo navigation on Riemannian manifolds. J. Differ. Geom. 66, $391-449$ (2004)

12. Xing, H: The geometric meaning of Randers metrics with isotropic S-curvature. Adv. Math. 34(6), 717-730 (2005)

13. Shen, ZM, Xing, H: On Randers metrics with isotropic S-curvature. Acta Math. Sin. 24, 789-796 (2008)

14. Ohta, S, Sturm, KT: Bochner-Weitzenbock formula and Li-Yau estimates on Finsler manifolds. Adv. Math. 252, $429-448$ (2014)

15. Milnor, J: A note on curvature and fundamental group. J. Differ. Geom. 2, 1-7 (1968)

16. Yang, YH: On the growth of the fundamental groups on nonpositive curvature manifolds. Bull. Aust. Math. Soc. 54, 483-487 (1996)

17. Xin, YL: Ricci curvature and fundamental group. Chin. Ann. Math., Ser. B 27(2), 113-120 (2006)

10.1186/1029-242X-2014-107

Cite this article as: Yin et al.: Some comparison theorems and their applications in Finsler geometry. Journal of Inequalities and Applications 2014, 2014:107

\section{Submit your manuscript to a SpringerOpen ${ }^{\odot}$ journal and benefit from:}

- Convenient online submission

Rigorous peer review

- Immediate publication on acceptance

Open access: articles freely available online

- High visibility within the field

- Retaining the copyright to your article 